\title{
Graphene For Biosensing Applications In Point-Of-Care Testing
}

\author{
Ioannis Prattis ${ }^{\mathrm{a}, 1}$, Ernestine Hui ${ }^{\mathrm{a}, \mathrm{b}}$, Patrik Gubeljak ${ }^{\mathrm{a}, 2}$, Gabriele S. Kaminski Schierle ${ }^{\mathrm{b}}$, \\ Antonio Lombardo ${ }^{\mathrm{c}, \mathrm{d}, 3}$, Luigi G. Occhipinti ${ }^{\mathrm{a}, 4}$ * \\ ${ }^{a}$ Cambridge Graphene Centre, Department of Engineering, University of Cambridge, $9 \mathrm{~J} J$ Thomson Avenue, \\ Cambridge CB3 OFA, United Kingdom \\ ${ }^{b}$ Department of Chemical Engineering and Biotechnology, University of Cambridge, Philippa Fawcett Drive, \\ Cambridge CB3 OAS, United Kingdom \\ ${ }^{c}$ London Centre for Nanotechnology, 17-19 Gordon Street, London WC1H 0AH \\ ${ }^{d}$ Department of Electronic \& Electrical Engineering - University College London - Torrington Place - London \\ WCIE 7JE
}

\begin{abstract}
Graphene and Graphene Related Materials (GRMs) exhibit a unique combination of electronic, optical and electrochemical properties which make them ideally suitable for ultra-sensitive and selective point-of-care testing (POCT) devices. POCT device-based applications in diagnostics require test results to be readily accessible anywhere to produce results within a short analysis timeframe. This review article provides a summary of methods and latest developments in the field of graphene and GRMs- based biosensing in POCT, and an overview of the main applications of the latter in nucleic acids and enzymatic biosensing, cell detection, and immunosensing. For each application, we discuss scientific and technological advances along with the remaining challenges, outlining future directions for widespread use of this technology in biomedical applications.
\end{abstract}

Keywords-Graphene, Biosensors, Point-of-Care, Biomolecules, Detection, Diagnostics

${ }^{l}$ https://orcid.org/0000-0002-6397-7201

${ }^{2}$ https://orcid.org/0000-0001-6955-419X

${ }^{3}$ https://orcid.org/0000-0003-3088-6458

${ }^{4} \mathrm{https}$ //orcid.org/0000-0002-9067-2534

*Correspondence: 1go23@cam.ac.uk (Luigi G. Occhipinti); www.occhipintigroup.com 


\section{Introduction to Graphene and GRMs Biosensors in POCT}

The development of novel and highly sensitive POCT devices has been the focus of research in the scientific community, due to the high impact that these devices may have for society. Over the recent years, and especially during the COVID-19 outbreak, remarkable advances have been made in the POCT field. Effective POCT devices are needed for rapid and reliable analytes' detection for disease diagnosis, monitoring, management, and for the prediction of treatment response $[1,2]$. The COVID-19 outbreak highlighted the urgency for commercially available POCT devices with tremendous impact on societies and economies. The molecular diagnostics area is projected to record higher growth rates over the next years, mainly due to the noteworthy advancements in biosensing technologies [3]. The World Health Organization (WHO) has developed the so called "Affordable, Sensitive, Specific, User-friendly, Rapid and Robust, Equipment-free, and Delivered- (ASSURED) criteria to be used as a benchmark for POCT products during their development phase [4].

Since the discovery of the unique electronic properties of graphene in 2004 [5], it has been intensively studied, revealing both interesting transport phenomena as well as potential for electronic application, including biosensing [6-8]. Thanks to the extremely high surface-area to volume ratio, graphene-based sensors are very sensitive to any changes in their environment [911]. This sensitivity makes them ideal for sensing applications, as -for example- a small amount of change in the concentration of an analyte in contact with graphene, modifies the electronic properties of the graphene enough to be measured [12]. Moreover, graphene and GRMs have additional advantages, as many of them can be functionalised with specific receptors or binding sites [13] to combine sensitivity and selectivity. The sensors then inherit the specificity from the functionalised groups, e.g., in the case of DNA detection the ability to detect specific sequences and identify single nucleotide mutations.

In this review, we present the latest advances in the field of graphene and GRMs biosensors that have been developed for nucleic acids biosensing, enzymatic biosensing and immunosensing, and have the potential to meet the ASSURED criteria for successful commercialisation in the near future. In addition, we focus on single layer graphene (SLG), few layers graphene (FLG), and the two most investigated chemical derivatives of graphene as enabling materials for applications in POCT: graphene oxide (GO) and reduced graphene oxide (rGO), as presented in (Box 1). Finally, we discuss the advances and challenges for each approach, and present our views on future 
directions in which the field is expected to grow and is likely to benefit from further research work and applications.

\section{RNA and DNA detection}

Over the last decade, there has been a significant interest in nucleic acid amplification and POCT due to their accurate and potentially rapid disease diagnosis capacity improving patient healthcare $[38,39]$. In comparison to the conventional detection approaches such as PCR, nucleic acid based POCTs can detect infectious agents in a much faster and convenient manner [40,41], thus acting as a potent tool for early diagnosis, detection, and monitoring of cancer [42, 43] and bacterial infections [44-48].

An ultra-sensitive graphene field effect transistor (GFET) functionalised with peptide nucleic acid (PNA) probes has been recently reported for RNA quantification [49]. The immobilisation of nucleic acid probes is done via p-p interaction on the surface of graphene. PNA probes have a neutral amine bond backbone that improves their binding specificity to DNA and RNA targets, and shortens their detection time [50,51]. The PNA biosensor had a Limit of Detection (LOD) which was three orders lower compared to DNA probes, and it was able to detect RNA targets at concentrations as low as $0.1 \mathrm{aM}$. In addition, the designed GFET biosensor was able to detect 100 aM RNA concentrations in human serum samples, promising results for future real-life applications. An alternative electrochemical-based sensing mechanism was presented by Gorgannezhad and colleagues [52] for the detection of $1 \mathrm{fM}$ ctRNA, which can serve as an alternative to tissue biopsies in ovarian cancer patients.

Numerous research articles have reported the development of graphene-based biosensors for RNA detection $[53,54]$ for which authors developed an isothermal platform for lung cancer-associated micro RNAs (miRNAs). The platform implemented GO-based fluorescence assays permit the rapid determination of single-base mismatches with a LOD of $0.87 \mathrm{fM}$. Graphene and GRMs POCT devices have also been applied to neurodegenerative diseases such as Alzheimer's Disease (AD). A graphene biosensor has been employed for the early detection of $\mathrm{AD}$ through the use of electrochemically reduced GO (ErGO) and gold nanowires to quantify serum miRNA-137, a reliable molecular biomarker for AD. The materials used permit improved sensitivity with a LOD of $1.7 \mathrm{fM}$ leading to better accuracy and reliability over the standard detection techniques - such as medical imaging, which poses problems such as bias from the physician [55]. This doxorubicin 
(Dox) intercalated screen-printed carbon electrode (SPCE) device has been tested using human serum; which highlights its potential clinical applications in the future [56]. Similar simple and cost effective SPCE devices based on the same materials have also been applied to PD, as shown by Figure 1, where the quantification of the plasma biomarker miRNA-195 to a LOD of $2.9 \mathrm{fM}$ could also be used as an early diagnostic technique in clinical settings [57].

Besides RNA detection, development of POC devices for DNA has also attracted significant interest by numerous researchers. A recent study by Kampeera and colleagues, [58] introduced a highly sensitive biosensor for Vibrio (V.) parahaemolyticus detection in raw seafood, one of the most important foodborne pathogens. The POCT platform was based on graphene SPCEs that are cheap and easy to fabricate at high volumes. Graphene-based SPCEs have larger electrochemical surfaces and higher electron mobility than other SPCEs, resulting in detection limit for V. parahaemolyticus of 0.3 Colony-forming unit (CFU) per $25 \mathrm{~g}$ of raw food within 45 minutes. A similar approach was followed by another research group to diagnose Tuberculosis, one of the most infectious diseases globally [59]. The study presents a graphene SPCE implemented on a portable potentiostat device, that is able to detect DNA fragments after loop-mediated isothermal amplification via label-free electrochemical analysis. The highly accurate sensor was able to detect up to $1 \mathrm{pg}$ of Mycobacterium tuberculosis DNA in less than 65 minutes.

The need for rapid, multiplexed, and affordable POCT devices for clinical diagnosis [60] led scientists to explore other graphene-based biosensing platforms [61]. Over the last few years, an impressive number of studies have been published, where GFET biosensors are used to detect DNA targets [62-64].

To enhance the sensitivity of GFET even further, crumpling of the graphene sheets over the conventional flat sheets was implemented as the biosensing element [65]. Due to the nanoscale deformation of crumpled graphene, the Debye length in the ionic solution is increased which results in decreased DNA charge screening. Furthermore, within the deformed regions, the formation of band gaps enables exponential changes of source-drain current by the presence of small charges [66]. These factors significantly enhance the sensitivity of the GFET biosensor down to zM DNA concentrations within 90 minutes. A similar approach was applied by Hwang and colleagues [66], where the crumpled GFET biosensor had a detection limit of $600 \mathrm{zM}$ compared to $2 \mathrm{pM}$ of flat graphene. 
In addition to all the above biosensing platforms for DNA detection, DNA sequencing is another fast-growing application. Knowing the exact sequence of the DNA can play a vital role in the diagnosis of diseases and disease treatment towards personalised medicine [67]. Current sequencing technologies do not offer fast and affordable methods applicable for POCT [68,69], however, due to the unique properties of graphene and GRMs they can have significant impact towards that direction [70-72]. One of the most well studied approaches is the development of graphene nanopores for DNA reading $[73,74]$. Graphene's thickness is smaller than the distance between 2 bases of oligonucleotides, allowing single base reading when it is used as a membrane for nanopore sequencing [75].

\section{Graphene based biosensors for protein detection}

Proteins - which can be found in blood or other tissues, are also widely used to predict, diagnose, and monitor of several diseases [76]. However, highly sensitive biosensors with LODs down to the femto- and picomolar concentrations, are required in order to use them as analytical biomarkers $[77,78]$.

Many studies have been published over the recent year, where graphene and GRMs are utilised in biosensing platforms to detect proteins that are released from cancer cells, or other cells as a response to cancer $[79,80]$. A novel electrochemical-based sensor with rGO, thionine and gold nanocomposites was developed for the detection of CA125 [81]. CA125 has been used as a protein biomarker for the early detection of ovarian cancer [82], and elevated concentrations in human serum have been related to lung and breast cancer too [83]. The fabrication of the immunosensor used screen printing technology on disposable cellulose paper, and the sensor was able to detect CA125 concentrations down to 0.01 Units per millilitre $(\mathrm{U} / \mathrm{mL})$ with a linear range from 0.01 to $200 \mathrm{U} / \mathrm{mL}$. Another interesting study was presented by Bharti and colleagues [84], who developed a carboxylated GO sensor for breast cancer. The aptasensor was immobilised with streptavidin to interact with the biotinylated aptamer for the MUC1 biomarker, a transmembrane glycoprotein on the surface of epithelial cells [85]. The biosensor was able to detect the MUC1 biomarker concentrations down to $0.79 \mathrm{fM}$ and displayed a great recovery range when serum samples were used.

Graphene-based biosensors have also been extensively studied for detecting viral proteins. Most recently, due to the COVID-19 outbreak, there was an urgent need for a rapid, accurate, and 
affordable POCT device for SARS-CoV-2 detection. Sea and colleagues [86] were amongst the first ones to develop a GFET biosensor functionalised with antibodies against the virus' spike protein, as shown by Figure 2. The device was able to detect protein concentrations of $1 \mathrm{fg} / \mathrm{mL}$ holding great potential for making rapid and accurate POCT devices.

\section{Enzyme-based graphene biosensors}

Enzymes are highly stable and can easily be manipulated, and thus are very useful in the design of POC biosensing platforms [87]. Enzymes can act as catalysts, performing chemical reactions after binding to specific substrates without being altered during the process [88]. The four most common types of enzymes used in biosensors are: amino oxidases, oxidoreductases, peroxidases, and polyphenol oxidases [89]; with oxidases and dehydrogenases being the two most encountered subclasses [90].

Optical and electrochemical bio-detection are the two main operational modes for enzyme-based biosensors [91]. Optical detection approaches are mainly applied for non-POCT oriented applications in healthcare, pharmaceuticals, and biomedical research, and are outside of the scope of this review. Enzyme-based graphene electrochemical biosensors due to their rapid, and easy mode of operation, and due to their label-free detection capacity.

Hajian and colleagues recently developed a CRISPR-Cas9-coated graphene-based biosensor for detecting DNA mutations related to Duchenne muscular dystrophy [92]. The sensor was sensitive enough to detect DNA targets of $1.7 \mathrm{fM}$ concentration within 15 minutes, as shown in Figure 3. The detection mechanism is based on a GFET, where the channel is functionalised with the CRISPR-Cas 9 complex. The enzymatic complex screens the genomic sequence until successful hybridisation of the single-guide RNA molecule to the target DNA motif. The hybridised complex changes the electrical properties of graphene resulting in an electrical output signal. This study has great potential in the POCT field due to the high specificity and label-free detection mode offered by the CRISPR-Cas9 enzymatic system. In addition, graphene's functionalisation with the enzymatic complex enables DNA detection without the need for reagents and bulky instrumentation.

Glucose monitoring biosensors are the most commercially successful POCT devices. An interesting approach of glucose sensing in sweat was reported by Xuan and colleagues [93], implementing GO on a flexible polyimide substrate. $\mathrm{rGO}$ and metal nanoparticles were applied to the working electrode and covered with glucose oxidase enzyme. The developed sensor had a great 
analytical performance with a detection range between $0.1-2.3 \mathrm{mM}$ within a 12 -seconds time frame. The advantages of the study are the development of a disposable, cost-effective, and responsive analytically performing enzyme-based glucose sensing patch. In addition, it works without the need of invasive procedures such as blood collection. Numerous other studies have developed glucose biosensors, implanting graphene as the sensing elements for improved performance [94-97]. A similar approach was developed for detecting 3-HB, a crucial diagnostic biomarker for life-threatening ketoacidosis [98]. The biosensors had a limit of detection of $1 \mu \mathrm{M}$, much higher compared to the commercially available kits for 3-HB detection.

Two other widely used enzymes for biosensing applications are laccase and horseradish peroxidase (HRP) [99]. These enzymes are cheaper compared to other ones and they can have a versatile range of applications. Laccase is an oxygen-reducing enzyme that can be applied to detect many analytes such as 17b-estradiol, phenols, and catechols [100,101], while HRP is used to determine the hydrogen peroxide levels in different samples [97]. In one of the most recent studies by Lopez Marzo and colleagues [102] the authors applied 3D printing to fabricate the electrode, before functionalising it with HRP to generate a direct electron transfer mechanism for hydrogen peroxide detection. This work has great potential for real sample analysis, since the direct electron transfer from the enzyme to the graphene electrodes eliminates the need for mediators. The previous studies capture a wide range of applications that graphene-based biosensors can be applied to measure enzymatic activity.

\section{Small Molecule Detection}

The ability of graphene sensors to detect small molecules such as gases $\left(\mathrm{NO}_{2}, \mathrm{NH}_{3}\right.$ etc.) and molecular ions (acetate, lactate etc.), relies on the low electronic noise and high sensitivity [103]. This can be used to detect and measure the presence of metabolic byproducts, such as acetone, down to $0.1 \mathrm{ppm}$ in the case of diabetic ketoacidosis [104] and 4ppm for nerve agent simulating compounds [105]. For POCT, there are several ways of using graphene small molecule sensors to develop clinical and diagnostic applications. Several adopted platforms are based on three-terminal devices in liquid electrolyte-gated field effect transistor or electrochemical transistor configurations, where both the active channel and the gate electrode are separated by the medium containing the molecules to be detected and exploit the selectivity of a bio-recognition elementscontaining membrane interposed between the channel or the gate electrode and the medium [106, 107]. In contrast all-solid-state transducers, are designed in a way that the active channel, the gate, 
and gate dielectric are all-solid state, exploiting a dependency of their electrical characteristics from the chemical or biochemical interactions with the surrounding environment. Li and colleagues [108] developed a novel highly accurate graphene-based ion sensitive field effect transistor (ISFET) for measuring the concentration of $\mathrm{K}+$ ions in different solutions, which can contribute significantly towards the onset prediction of myocardial infractions and seizure. The graphene-based ISFET sensor had a wide detection range of ionic concentrations $(1 \mu \mathrm{M}-2 \mathrm{mM})$ with comparable sensitive to Si-based ISFET devices ( $>60 \mathrm{mV} /$ decade).

Most often graphene sensors for small molecules are chemiresistive, i.e., their conductivity changes when there is a chemical change in their environment [109], but the change in their optical properties can also be used to form "litmus paper" like composites [110], or to make sensors to be fluorescent [111] or display increased absorption of light [112].

An emerging diagnostic technique is the characterisation of volatile organic compounds (VOCs) from a patient's breath, permitting for early detection and classification of diseases such as cancer [113] and infectious diseases [114]. In total, more than 2600 compounds have been identified as biomarkers, such as NO for asthma, n-pentane for heart disease [115], $\mathrm{NH}_{3}$ for kidney malfunction [116], to name a few. An example of such a biomarker sensor for $\mathrm{NH}_{3}$ was reported by Bhardwaj and colleagues [117], with a detection limit of $0.6 \mathrm{ppm}$ and range of 1-100 ppm, sufficient for POC VOC detection.

\section{Cancer Cells and Bacterial Detection}

Whole cells have also been classified using graphene and graphene-related material POCT devices [118]. An ultrasensitive electrochemical cytosensor, which uses graphene, gold nanoparticles, and HRP-conjugated trastuzumab antibody hybrid structures as a nanoprobe, has a low LOD of 20 cells/mL, and has been used for the detection of human prostate metastatic cancer cells [119]. HeLa cells and MCF-7 breast cancer cells have also been detected using impedimetric biosensors [120].

Another promising POCT for clinical applications is the sensitive and rapid detection of cancer cells using graphene functionalised magnetic microbeads and the photothermal effect. It works on the basis of modifying GO with antiepithelial cell adhesion molecule antibodies and modifying the magnetic microbeads with anti-immunoglobulin. The modified GO and magnetic microbeads 
are mixed, incubated with 4T1 breast cancer cells, filtered using a membrane, and separated magnetically before being detected using a laser and an infrared camera. The temperature variation before and after laser irradiation was used to quantify the number of cancer cells the magnetic beads attached to. Further tests were carried out on the detection of 4T1 cells in human blood, displaying a LOD of 100 cells/mL [121].

Escherichia (E.) coli contaminated drinking water has detrimental effects on humans, especially if there is a delay of treatment after the appearance of initial symptoms. Therefore, early detection and real-time monitoring is essential to combat the life-threatening effects of the Shiga toxins released by strains of E. coli. Current methods such as PCR and colony-counting methods do not possess the sensitivity required to detect low concentrations of bacteria. The sensitivity of this device is of the utmost importance, as bacterium would already be able to cause disease. In addition, the current devices display high background signals and poor specificity making the diagnostic costs increase as a result of the equipment and training required. This rGO-based field effect transistor (FET), as shown in Figure 4, has been modified with antibodies against E. coli to detect individual bacteria. The device was adapted to detect various strains of E. coli through the use of a generic antibody. It works on the basis of depositing an rGO sheet on a gold/silicon dioxide electrode surface. As there is a negative charge around the E. coli membrane, when it binds with the rGO, the electrical conductivity of rGO in the FET will be modulated. This low cost and disposable device is capable of rapidly detecting individual E. coli in a river water sample - making it ideal for mass production and large volume testing [122].

A similar device has been developed where a monolayer graphene nanostructure was functionalised with antibodies to capture pathogenic bacteria with a sensitivity of $10-100$ cells $/ \mathrm{mL}$. The capture process then generates a non-Faradaic electrochemical signal specific to that of E. coli. This label-free method works on the basis of dielectric changes of capacitance and impedance, however, the trade off to the sensitivity provided by the monolayer graphene is the cost and complexity in the production of a defect-free layer [123].

A hybrid microfluidic and FET system has also been demonstrated as a POCT device for E. coli. This portable and real-time monitoring hybrid uses a graphene micropatterned FET conjugated with dual antibiotic bioprobes for the early detection of E. coli. A low LOD of $10^{0}$ colony forming units/mL (1-9 CFU/mL) was obtained. CVD graphene was used to fabricate the FET and a polydimethylsiloxane (PDMS) microfluidic chip was then attached to the bioprobe conjugated 
GFET. Four types of bacterial species - E. coli, Salmonella enterica, Staphylococcus aureus, and Enterococcus faecium were to be studied. E. coli and Salmonella enterica were detected through using specific vancomycin bioprobes for rapid detection, and magainin I bioprobes were used for the detection of Staphylococcus aureus and Enterococcus faecium [124].

\section{Concluding remarks and future perspectives}

The field of graphene and GRMs biosensors has been significantly expanding over the past years in different areas of research and was driven by the most recent advances in the fundamental understanding of the material properties, the engineering of their scalable production methods, and the functionalisation techniques for their application in biology, medicine, and chemistry. Over the past decade, several breakthroughs in the field have been achieved, with pioneering research work on detection methods and novel 2D materials paving the way towards the development of highly accurate and sensitive biosensing platforms to detect a broad range of disease biomarkers - as summarised in (Table 1).

When focusing on future POCT devices key properties of 2D materials that determine their suitability for rapid and accurate diagnostic platforms include their biocompatibility, optical transparency, and large-scale and high-quality manufacturing capabilities. Both CVD growth of graphene and GRMs, microfluidisation and electrochemical-based production processes are well placed to obtain suitable material at an industrial scale [28, 32]. However, there are still challenges needed to be overcome (see Outstanding Questions) for successful commercialisation of graphenebased POCT.

The demand for reliable and affordable POCT devices alongside easy-to-use operational systems for diagnostic applications is driving the research of novel biomarkers that can be detected via GRMs-based biosensors from multiple biological sources and with a minimum amount of sample material, along with simplified sample preparation methods. Achieving ultimate sensitivity of graphene and GRMs-based biosensors to current and future biomarkers in cells, proteins, RNA, DNA along with the high specificity to the same biomarkers means that future POCT devices will enable highly efficient "sample-in answer-out" diagnostic platforms for point-of-care applications with high-throughput, small volumes and cost of reagents and minimal and without the occurrence of false positives / false negatives $[125,126]$. 
Additionally, the emergence of electrical biosensing technologies enabling accurate and real-time electrical instead of conventional optical-based (particularly fluorescence) detection methods will allow an entirely new set of advanced signal processing and data analysis techniques to be applied along with existing bioinformatics methods for point-of-care diagnostics of cancer, cardiovascular or neurological diseases as well as for the diagnostics and screening of rapidly spreading infectious diseases, including those resulting in global pandemics, where time-to-answer and cost of the POCT device plays a critical role in determining the effectiveness of the response from various government agencies and healthcare providers. When we compare the technologies presented in this review with conventional POCT devices currently adopted in clinical laboratory practice such as those based on qPCR, there is a clear opportunity to achieve the following improvements; at least 50x reduction in the volume and therefore cost of reagents, including PCR master mix, primers, fluorescence probes and enzymes, which result in approximately $60 \%$ reduction to the overall cost per test for the proposed electrical detection. In addition, the use of emerging sensing technologies presented in this review has the potential to achieve about $3 \mathrm{x}$ improved sensitivity for the detection of protein biomarkers as compared to the current POCT devices making the approach interesting for immunoassay applications and new areas of clinical and non-clinical research.

Global megatrends such as the ageing population alongside the proliferation of the Internet of Things and artificial intelligence, are only a few of the additional context- related drivers for the future growth and broadening of graphene and GRMs-based POCT devices. Combined with their easy integration with existing technologies, graphene and GRMs materials will play a key role for applications in personalised diagnostic and integrated health monitoring systems $[127,128]$.

\section{Declaration}

The authors declare no conflict of interest. 


\section{Figures}
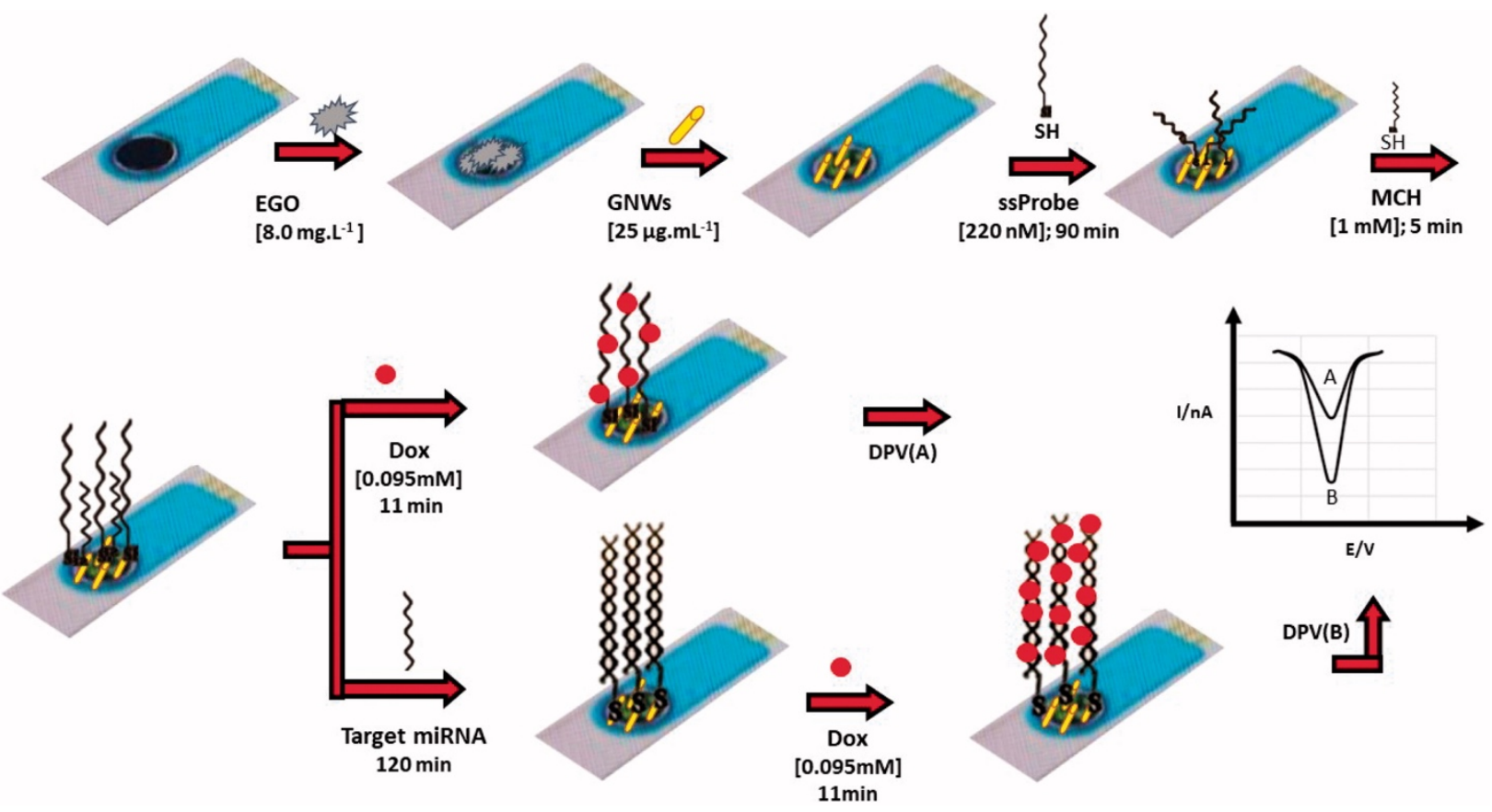

Figure 1: Figure demonstrating how a SPCE POCT device detects miR-195, a plasma biomarker for the early detection of PD. Reproduced with permission from [57].

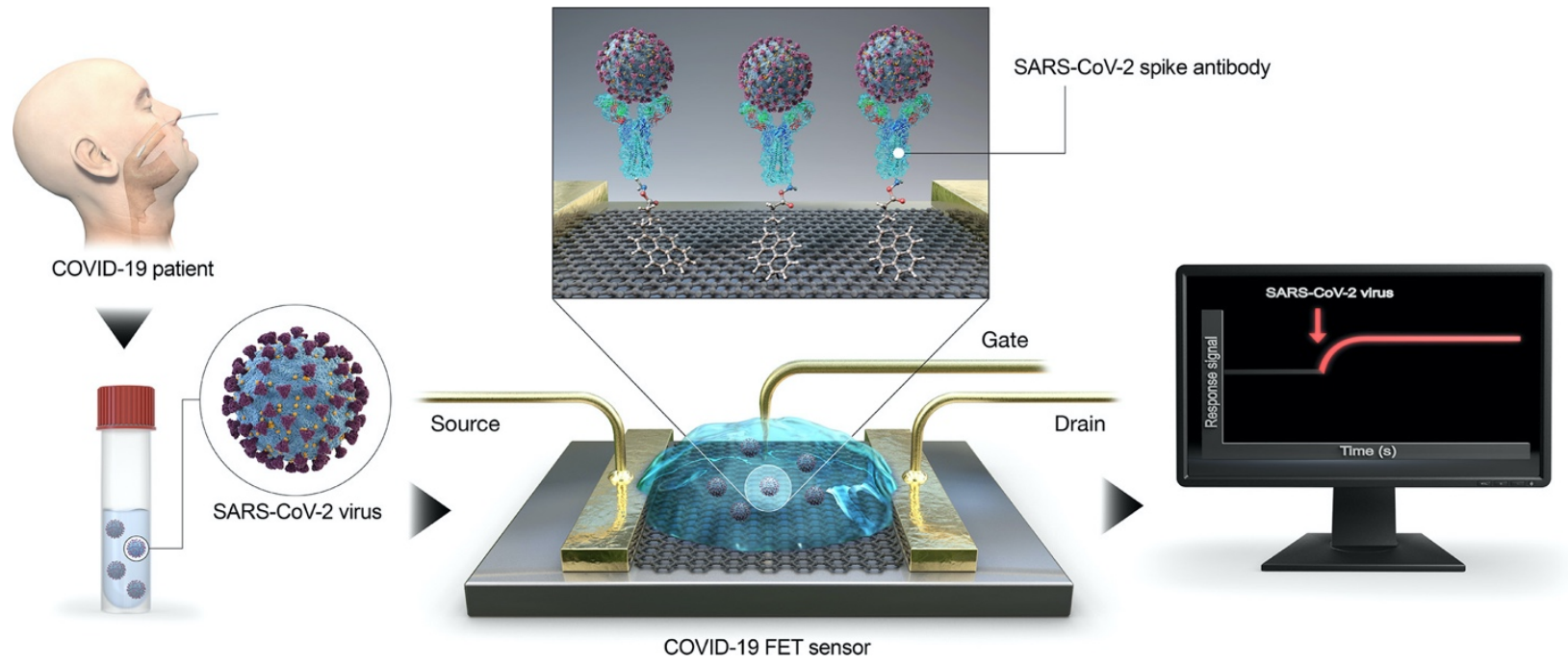

Figure 2: Schematic representation of the GFET biosensor developed by Sea and colleagues for detecting the SARS-CoV-2 spike protein. Reproduced with permission from [86]. 


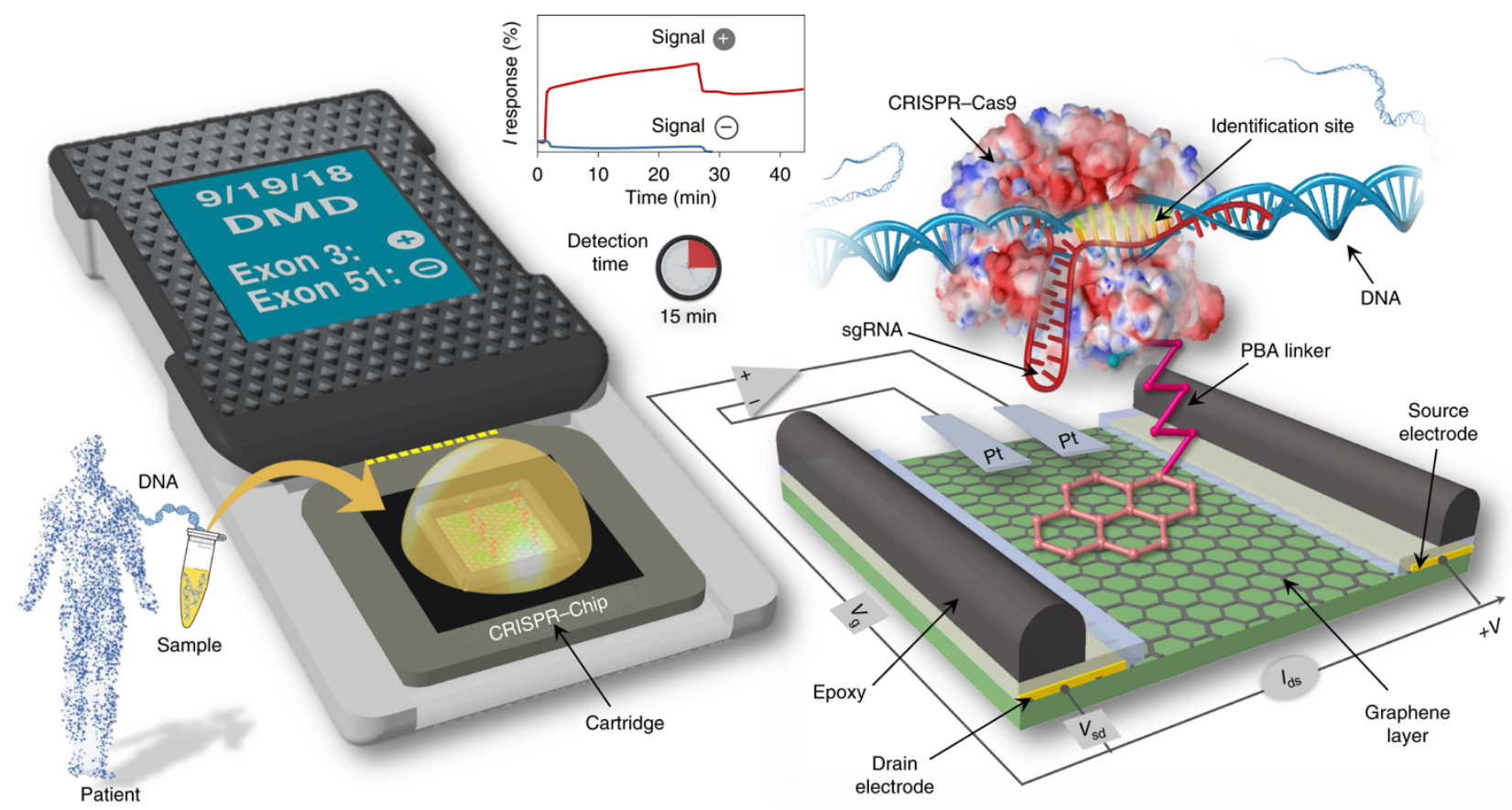

Figure 3: CRISPR-Cas9 biosensor developed by Hajian and colleagues for the detection of unamplified DNA targets in less than 15 min. Reproduced with permission [92].

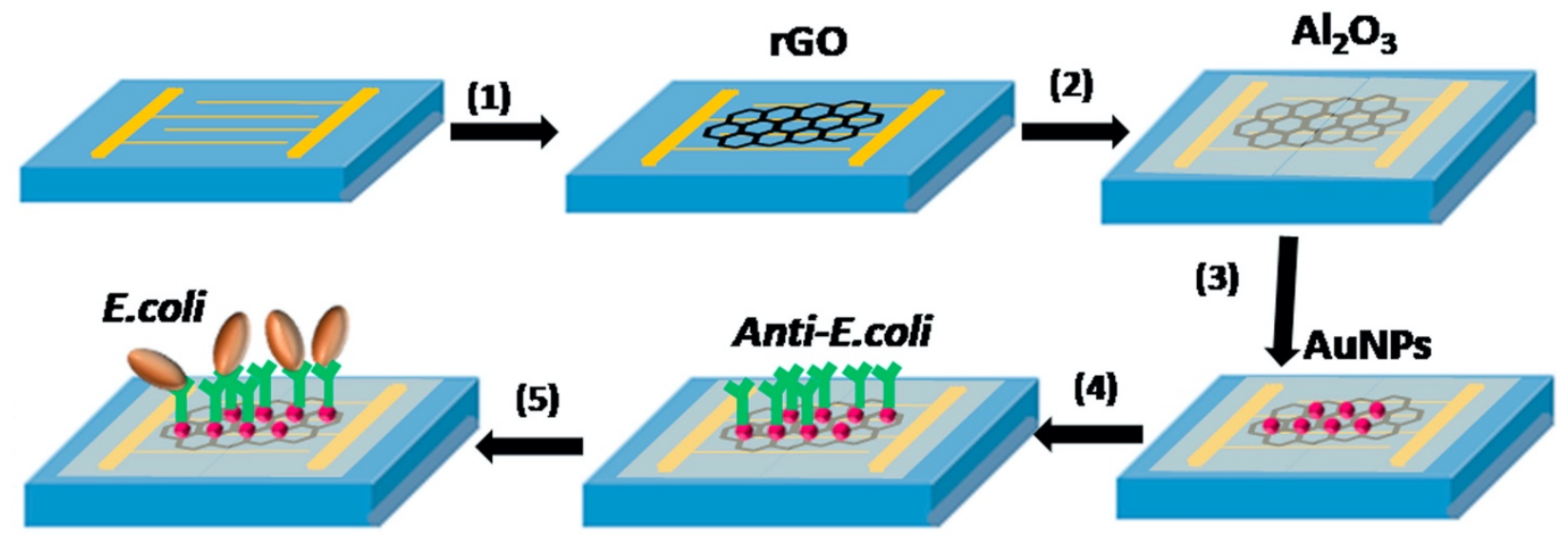

Figure 4: Figure demonstrating the fabrication of the rGO FET sensor. (1) Self-assembly of rGO followed by thermal reduction, (2) Deposition of atomic layer of $\mathrm{Al}_{2} \mathrm{O}_{3}$, (3) Deposition of $\mathrm{AuNPs}$, (4) Immobilisation of Anti-E. coli, and (5) E. coli sensing. Reproduced with permission from [122]. 


\section{Box 1: Production methods of graphene and GRMs}

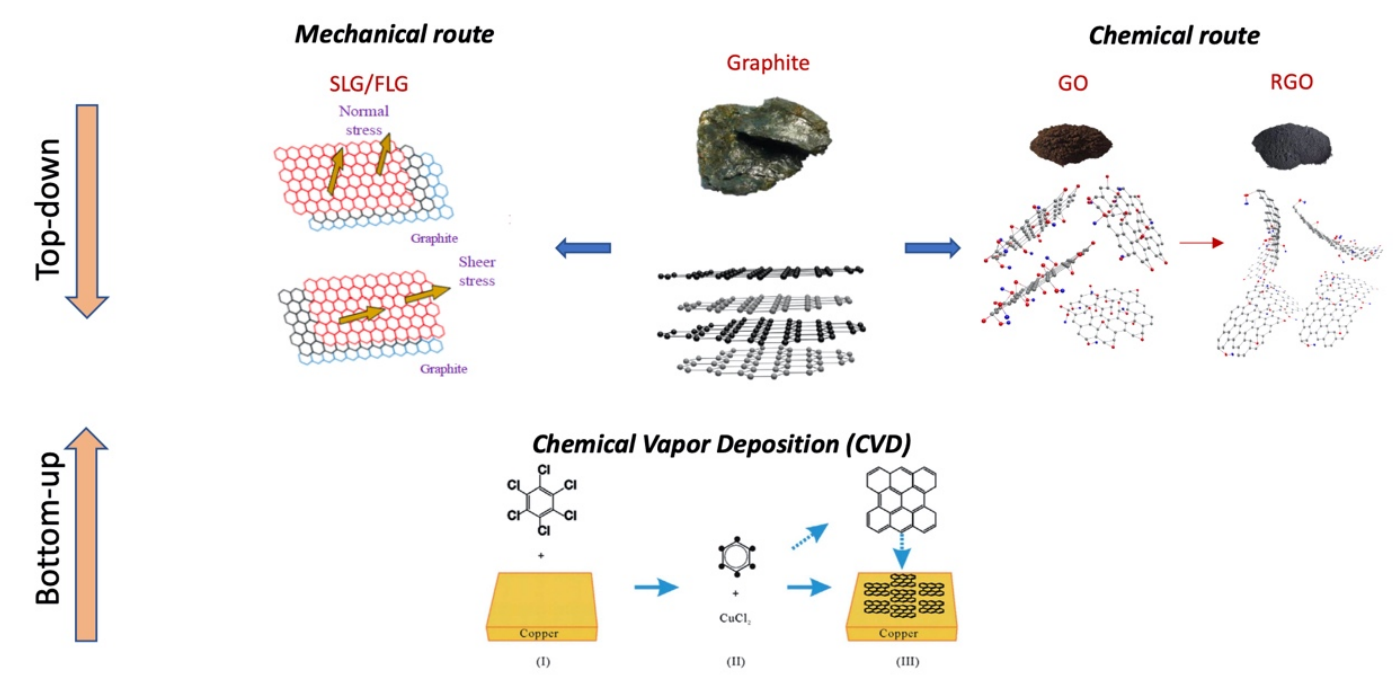

Figure I - Figure demonstrating different graphene production routes from graphite. These include top-down and bottom-up approaches (adapted with permission from [37]).

Over the last fifteen years, several production methods have been developed for graphene and its chemical derivatives $[14,15]$. This review focuses only on scalable methods, which we classify as either top down or bottom up. The first class includes methods where single or few layers of graphene are extracted from graphite, while the latter refers to graphene synthesis from a suitable precursor. As production and processing of graphene and GRMs often determine their properties, we will consider separately large area single layer graphene (SLG) grown via chemical vapour deposition (CVD) and graphene suspensions obtained from liquid phase exfoliation (LPE) [16].

\subsection{Mechanical and liquid-phase exfoliation of graphene and GRMs (top-down)}

Mechanical exfoliation from graphite was the pioneering method used to produce graphene. This top-down approach works on the basis of the repeated peeling of graphite using an adhesive tape [5]. Due to weak inter-layer van der Waals forces, the magnitude of force provided by the tape is sufficient to split the graphite down to a single layer [17]. Despite the affordability and ease, this process is not suitable for large scale processes as it produces irregular and small flakes (lateral size $<1 \mathrm{~mm}$ ) at a low yield [14, 18-20]. LPE is another top-down technique, employing ultrasound or shear forces to exfoliate graphite to obtain individual graphene layers in a liquid environment. LPE is easily scalable, however, the difficulty lies in the stabilisation of the solution to prevent reaggregation $[14,18]$.

\subsection{Graphene Oxide}

GO is obtained via the LPE of oxidized graphite. It is cost effective and industrially scalable; with production being on the scale of tons [21]. These multiple stacked layers of GO consist of oxygen bonded to graphene on its basal plane. The oxygenated groups allow for properties such as dispersibility in water without surfactants [22-24]. Despite the scalability, the stability of the 
colloidal dispersions and the yield obtained is an issue [17,25]. GO can also be used to fabricate graphene quantum dots (GQD), which can be prepared hydrothermally or electrochemically [26].

\subsection{Reduced Graphene Oxide}

Reduction of GO via thermal, photocatalytic, or chemical methods result in rGO, which retains the properties of graphene and benefit from the production methods of GO [27]. The challenges are due to the presence of defective groups as a result of the GO production method. There is also a tendency to form aggregates, as the reduction process removes the oxygen groups that contribute to the stability of GO in water [28]. Recently, rGO production has been focused on non-toxic and low energy consumption processes ranging from microbial reduction $[28,29]$ to electrochemical reduction $[30,31]$.

\subsection{Chemical vapour deposition (bottom-up)}

The most widely used bottom-up method to obtain graphene is CVD. This process exposes transition metal substrates to hydrocarbon gasses at high temperatures to introduce carbon $[16,17$, $20,32,33]$. It is a self-limiting process and the size of the films produced is only limited by the reactor size, with wafer-scale CVD systems being commercially available. However, device fabrication requires transfer from the metal foil where it is grown to an insulating substrate, introducing impurities and defects. However, CVD is one of the most promising routes to obtaining large-scale and high quality SLG, and can also be used to prepare other GRMs, such as graphene nanoplatelets (GNP) [32, 34-36]. 
Table 1

Table 1: State-Of-The-Art POCT Devices Summary

\begin{tabular}{|c|c|c|c|c|c|}
\hline Material & Functionalisation & Device Architecture & Analyte & Sensitivity/ LOD & Reference \\
\hline ErGO & Gold nanowires & $\begin{array}{l}\text { Dox intercalated } \\
\text { SPCE }\end{array}$ & $\begin{array}{l}\text { Serum } \\
\text { miRNA-137 }\end{array}$ & $1.7 \mathrm{fM}$ & {$[56]$} \\
\hline ErGO & Gold nanowires & SPCE & $\begin{array}{l}\text { Plasma } \\
\text { miRNA-195 }\end{array}$ & $2.9 \mathrm{fM}$ & [57] \\
\hline GO & $\begin{array}{l}\text { Antiepithelial cell } \\
\text { adhesion molecule } \\
\text { and magnetic mi- } \\
\text { crobeads with anti- } \\
\text { immunoglobulin }\end{array}$ & $\begin{array}{l}\text { Photothermal } \\
\text { detection }\end{array}$ & $\begin{array}{l}4 \mathrm{~T} 1 \text { breast } \\
\text { cancer cells }\end{array}$ & 100 cells $/ \mathrm{mL}$ & {$[121]$} \\
\hline rGO & Gold nanoparticles & FET & E. coli & $10^{3}-10^{5} \mathrm{CFU} / \mathrm{mL}$ & {$[122]$} \\
\hline $\begin{array}{l}\text { GNP and } \\
\text { graphene }\end{array}$ & $\begin{array}{l}\text { Anti-E. coli } \\
\text { O157:H }\end{array}$ & Electrical biosensor & E. coli & $10-100$ cells $/ \mathrm{mL}$ & {$[123]$} \\
\hline $\begin{array}{l}\text { Graphene } \\
\text { quantum } \\
\text { dots }\end{array}$ & E. coli antibody & $\begin{array}{l}\text { Electrogenerated } \\
\text { chemiluminescence }\end{array}$ & E. coli & $5 \mathrm{CFU} / \mathrm{mL}$ & [129] \\
\hline Graphene & $\begin{array}{l}\text { Antibiotic } \\
\text { bioprobes }\end{array}$ & Microfluidic/FET & E. coli & $10^{0}-10^{1} \mathrm{CFU} / \mathrm{mL}$ & [124] \\
\hline Graphene & PNA & FET & RNA & $100 \mathrm{aM}$ & [49] \\
\hline GO & $\mathrm{Fe}_{2} \mathrm{O}_{3}$ nanoparticles & SPCE & $\begin{array}{l}\text { Ovarian } \\
\text { cancer } \\
\text { ctRNA }\end{array}$ & $1 \mathrm{fM}$ & {$[52]$} \\
\hline GO & DNA & Fluorescence & $\begin{array}{l}\text { Lung cancer } \\
\text { miRNA }\end{array}$ & $0.87 \mathrm{fM}$ & [53] \\
\hline Graphene & DNA & FET & DNA & $5 \mathrm{fM}$ & [65] \\
\hline rGO & Aptamer & SPCE & $\begin{array}{l}\text { Ovarian } \\
\text { cancer } \\
\text { biomarker } \\
\text { CA125 }\end{array}$ & $0.01 \mathrm{U} / \mathrm{mL}$ & [81] \\
\hline rGO & Aptamer & FET & $\begin{array}{l}\text { HPV-16 } \\
\text { E7 protein }\end{array}$ & $1.75 \mathrm{nM}$ & {$[130]$} \\
\hline Graphene & Antibody & FET & $\begin{array}{l}\text { SARS-CoV- } \\
2 \text { Spike } \\
\text { protein }\end{array}$ & $1 \mathrm{fg} / \mathrm{mL}$ & [86] \\
\hline Graphene & CRISPR & FET & $\begin{array}{l}\text { DNA } \\
\text { mutations } \\
\text { for } \\
\text { Duchenne } \\
\text { muscular } \\
\text { distrophy }\end{array}$ & $1.7 \mathrm{fM}$ & [92] \\
\hline rGO & Gold nanoparticles & $\begin{array}{l}\text { Electrochemical } \\
\text { electrode }\end{array}$ & Glucose & $0.1 \mathrm{mM}$ & [93] \\
\hline Graphene & Folic acid & $\begin{array}{l}\text { Surface Plasmon } \\
\text { Resonance }\end{array}$ & FAP & $5 \mathrm{fM}$ & [111] \\
\hline $\begin{array}{l}\text { Metal- } \\
\text { Organic } \\
\text { composite } \\
\text { with } \\
\text { graphene }\end{array}$ & None & Chemiresistive & $\mathrm{NH}_{3}$ & $0.36 \mathrm{ppm}$ & [117] \\
\hline
\end{tabular}




\section{Glossary}

Chemical vapour deposition (CVD) is a deposition process where gaseous reactants are deposited onto a substrate to generate a thin film. CVD is used to grow large area graphene films, normally using methane as precursor and $\mathrm{Cu}$ as catalytic substrate.

Field Effect transistor is a three-terminal (source, drain and gate) semiconductor device that utilises the electric field to control the flow of current in the channel.

Graphene field effect transistor is a FET device having graphene as channel connecting source and drain terminals. A voltage applied to the gate terminal modulates the conductivity of the channel. The graphene can be functionalised with receptors for detection of specific analytes.

Graphene nanoplatelets are flakes consisting of few graphene layers.

Graphene oxide is the oxidised form of graphene with several oxygen-containing functional groups such as carbonyl, carboxyl, epoxide, and hydroxyl. Due to the oxygen presence in its lattice, GO is not conductive.

Graphene quantum dots are zero dimensional carbon-based nanoparticles and are cut fragments from graphene sheets with lateral dimensions less than $100 \mathrm{~nm}$.

Ion sensitive field effect transistors are FET devices that electrically measure the ion concentrations in solutions, which act as the gate electrode. The concentration of the negatively or positively charged molecules into solution controls the current flow through the channel.

Liquid phase exfoliation (LPE) is a process used to obtain dispersions of graphene, few layers graphene (FLG) flakes and graphene nanoplatelets in a suitable solvent. LPE typically uses ultrasounds or shear forces to break the bonds between layers in bulk graphite. LPE is a low-cost, scalable technique used to produce graphene dispersions useful for printing or composite materials.

Reduced graphene oxide is the material resulting from reduction of graphene oxide. As the reduced material is typically defective and does not possess the same electrical characteristic of graphene, the term rGO is used to differentiate from graphene. 


\section{References}

1 Jia, Y. et al. (2018) Paper-based graphene oxide biosensor coupled with smartphone for the quantification of glucose in oral fluid. Biomed. Microdevices 20, 89

2 Zhang, Z. et al. (2019) A versatile, cost-effective, and flexible wearable biosensor for in situ and ex situ sweat analysis, and personalized nutrition assessment. Lab Chip 19, 3448-3460

3 Buyer, R. (2018) Point-of-Care/Rapid Diagnostics Market by Testing, Platform, Mode, End-User - Global Forecast to 2022,

4 Peeling, R.W. et al. Rapid tests for sexually transmitted infections (STIs): The way forward. , Sexually Transmitted Infections, 82. (2006)

5 Novoselov, K.S. et al. (2004) Electric field in atomically thin carbon films. Science (80-. ). 306, 666-669

6 Yafia, M. et al. (2020) Low-Cost Graphene-Based Digital Microfluidic System. Micromachines 11,880

7 Piccinini, E. et al. (2017) Enzyme-polyelectrolyte multilayer assemblies on reduced graphene oxide field-effect transistors for biosensing applications. Biosens. Bioelectron. 92, 661-667

8 Park, S.J. et al. (2012) Ultrasensitive Flexible Graphene Based Field-Effect Transistor (FET)-Type Bioelectronic Nose. DOI: 10.1021/n1301714x

9 Hill, E.W. et al. (2011) Graphene Sensors. IEEE Sens. J. 11, 3161-3170

10 Mali, K.S. et al. (2015) Nanostructuring graphene for controlled and reproducible functionalization. Nanoscale 7, 1566-1585

11 Goniszewski, S. et al. (2016) Correlation of p-doping in CVD Graphene with Substrate Surface Charges. Sci. Rep. 6,

$12 \mathrm{Ni}$, J. et al. (2020) Adsorption of small gas molecules on transition metal (Fe, $\mathrm{Ni}$ and Co, $\mathrm{Cu}$ ) doped graphene: A systematic DFT study. Phys. E Low-Dimensional Syst. Nanostructures 116,

13 Georgakilas, V. et al. (2012) Functionalization of Graphene: Covalent and Non-Covalent Approaches, Derivatives and Applications. Chem. Rev. 112, 6156-6214

14 Bonaccorso, F. et al. Production and processing of graphene and 2d crystals., Materials Today, 15. 01-Dec-(2012), Elsevier B.V., 564-589

15 Backes, C. et al. (2020) Production and processing of graphene and related materials. $2 D$ Mater. 7, 022001

16 Wasfi, A. et al. Graphene-based nanopore approaches for DNA sequencing: A literature review. , Biosensors and Bioelectronics, 119. 15-Nov-(2018), Elsevier Ltd, 191-203

17 Soldano, C. et al. Production, properties and potential of graphene., Carbon, 48. 01-Jul(2010), Pergamon, 2127-2150

18 Ferrari, A.C. et al. (2015) Science and technology roadmap for graphene, related twodimensional crystals, and hybrid systems. Nanoscale 7, 4598-4810

19 Karagiannidis, P.G. et al. (2017) Microfluidization of Graphite and Formulation of Graphene-Based Conductive Inks. ACS Nano 11, 2742-2755

20 Kumar, S. et al. (2019) Electrochemical Sensors and Biosensors Based on Graphene Functionalized with Metal Oxide Nanostructures for Healthcare Applications. ChemistrySelect 4, 5322-5337

21 Zhu, Y. et al. (2010) Graphene and graphene oxide: Synthesis, properties, and applications. Adv. Mater. 22, 3906-3924

22 Smith, A.T. et al. (2019) Synthesis, properties, and applications of graphene oxide/reduced graphene oxide and their nanocomposites. Nano Mater. Sci. 1, 31-47 
23 Taniselass, S. et al. Graphene-based electrochemical biosensors for monitoring noncommunicable disease biomarkers. , Biosensors and Bioelectronics, 130. 01-Apr-(2019), Elsevier Ltd, 276-292

24 Brisebois, P.P. and Siaj, M. Harvesting graphene oxide-years 1859 to 2019: A review of its structure, synthesis, properties and exfoliation., Journal of Materials Chemistry C, 8. 06-Feb(2020), Royal Society of Chemistry, 1517-1547

25 Chen, D. et al. Graphene oxide: Preparation, functionalization, and electrochemical applications. , Chemical Reviews, 112. 14-Nov-(2012), American Chemical Society, 6027-6053

26 Liu, R. et al. (2011) Bottom-up fabrication of photoluminescent graphene quantum dots with uniform morphology. J. Am. Chem. Soc. 133, 15221-15223

27 Tarcan, R. et al. Reduced graphene oxide today., Journal of Materials Chemistry C, 8. 30Jan-(2020), Royal Society of Chemistry, 1198-1224

28 Feng, J. et al. Synthetic routes of the reduced graphene oxide., Chemical Papers, 74. 01Nov-(2020), Springer, 3767-3783

29 Luo, D. et al. (2011) Evaluation criteria for reduced graphene oxide. J. Phys. Chem. C 115, $11327-11335$

30 Peng, X.Y. et al. (2011) Synthesis of electrochemically-reduced graphene oxide film with controllable size and thickness and its use in supercapacitor. Carbon N. Y. 49, 3488-3496

31 Marrani, A.G. et al. (2018) Integration of graphene onto silicon through electrochemical reduction of graphene oxide layers in non-aqueous medium. Appl. Surf. Sci. 445, 404-414

32 Bae, S. et al. (2010) Roll-to-roll production of 30-inch graphene films for transparent electrodes. Nat. Nanotechnol. 5, 574-578

$33 \mathrm{Li}$, X. et al. (2009) Transfer of large-area graphene films for high-performance transparent conductive electrodes. Nano Lett. 9, 4359-4363

$34 \mathrm{Li}$, X. et al. (2009) Large-area synthesis of high-quality and uniform graphene films on copper foils. Science (80-. ). 324, 1312-1314

35 Liu, Y. et al. (2012) Biological and chemical sensors based on graphene materials. Chem. Soc. Rev. 41, 2283-2307

36 Awadallah, A.E. et al. (2017) Facile and large-scale synthesis of high quality few-layered graphene nano-platelets via methane decomposition over unsupported iron family catalysts. Mater. Chem. Phys. 191, 75-85

37 Ng, L.W.T. et al. (2019) Structures, Properties and Applications of 2D Materials. In Printing of Graphene and Related 2D Materials pp. 19-51, Springer International Publishing

38 Fan, X. et al. (2018) A graphene-based biosensor for detecting microRNA with augmented sensitivity through helicase-assisted signal amplification of hybridization chain reaction. Sensors Actuators, B Chem. 255, 1582-1586

39 Zhu, G. and Qiao, M. Nucleic acid amplification by a transparent graphene Visual-PCR chip and a disposable thermocycler. , bioRxiv. 07-Aug-(2019) , bioRxiv, 724245

40 Chen, J.Y. et al. (2019) Ultrasensitive Electrochemical Biosensor Developed by Probe Lengthening for Detection of Genomic DNA in Human Serum. Anal. Chem. 91, 4552-4558

41 Lu, W. et al. (2016) Quantitative Detection of MicroRNA in One Step via Next Generation Magnetic Relaxation Switch Sensing. ACS Nano 10, 6685-6692

42 Liu, H. et al. (2017) In Situ Hot-Spot Assembly as a General Strategy for Probing Single Biomolecules. Anal. Chem. 89, 4776-4780

43 Zhou, W. et al. (2017) Building Electromagnetic Hot Spots in Living Cells via TargetTriggered Nanoparticle Dimerization. ACS Nano 11, 3532-3541 
Jaiswal, N. et al. (2020) An impedimetric biosensor based on electrophoretically assembled $\mathrm{ZnO}$ nanorods and carboxylated graphene nanoflakes on an indium tin oxide electrode for detection of the DNA of Escherichia coli O157:H7. Microchim. Acta 187, 1

45

Dai, G. et al. (2019) Electrochemical determination of Salmonella typhimurium by using aptamer-loaded gold nanoparticles and a composite prepared from a metal-organic framework (type UiO-67) and graphene. Microchim. Acta 186, 620

46 Pourmadadi, M. et al. (2019) A glassy carbon electrode modified with reduced graphene oxide and gold nanoparticles for electrochemical aptasensing of lipopolysaccharides from Escherichia coli bacteria. Microchim. Acta 186, 787

47 Farzin, L. et al. (2020) Electrochemical genosensor based on carbon nanotube/amine-ionic liquid functionalized reduced graphene oxide nanoplatform for detection of human papillomavirus (HPV16)-related head and neck cancer. J. Pharm. Biomed. Anal. 179, 112989

48 Li, J. et al. (2020) Sensitive electrochemical detection of hepatitis C virus subtype based on nucleotides assisted magnetic reduced graphene oxide-copper nano-composite. Electrochem. commun. 110, 106601

49 Tian, M. et al. (2020) Highly-sensitive graphene field effect transistor biosensor using PNA and DNA probes for RNA detection. Appl. Surf. Sci. 527, 146839

50 Movilli, J. et al. (2018) Control of Probe Density at DNA Biosensor Surfaces Using Poly(1 -lysine) with Appended Reactive Groups. Bioconjug. Chem. 29, 4110-4118

51 Mat Zaid, M.H. et al. (2017) PNA biosensor based on reduced graphene oxide/water soluble quantum dots for the detection of Mycobacterium tuberculosis. Sensors Actuators, B Chem. 241, 1024-1034

52 Gorgannezhad, L. et al. (2018) Detection of FGFR2 : FAM76A Fusion Gene in Circulating Tumor RNA Based on Catalytic Signal Amplification of Graphene Oxide-loaded Magnetic Nanoparticles. Electroanalysis 30, 2293-2301

53 Khoothiam, K. et al. (2019) Ultrasensitive detection of lung cancer-associated miRNAs by multiple primer-mediated rolling circle amplification coupled with a graphene oxide fluorescencebased (MPRCA-GO) sensor. Analyst 144, 4180-4187

54 Gao, J. et al. (2020) Ultrasensitive Label-free MiRNA Sensing Based on a Flexible Graphene Field-Effect Transistor without Functionalization. ACS Appl. Electron. Mater. 2, 10901098

55 Teipel, S. et al. Multimodal imaging in Alzheimer's disease: Validity and usefulness for early detection., The Lancet Neurology, 14. 01-Oct-(2015) , Elsevier, 1037-1053

56 Azimzadeh, M. et al. (2017) Early detection of Alzheimer's disease using a biosensor based on electrochemically-reduced graphene oxide and gold nanowires for the quantification of serum microRNA-137. RSC Adv. 7, 55709-55719

57 Aghili, Z. et al. (2018) A highly sensitive miR-195 nanobiosensor for early detection of Parkinson's disease. Artif. Cells, Nanomedicine, Biotechnol. 46, 32-40

58 Kampeera, J. et al. (2019) Point-of-care rapid detection of Vibrio parahaemolyticus in seafood using loop-mediated isothermal amplification and graphene-based screen-printed electrochemical sensor. Biosens. Bioelectron. 132, 271-278

59 Jaroenram, W. et al. (2020) Graphene-based electrochemical genosensor incorporated loop-mediated isothermal amplification for rapid on-site detection of Mycobacterium tuberculosis. J. Pharm. Biomed. Anal. 186, 113333 
60 Soler, M. et al. (2020) How Nanophotonic Label-Free Biosensors Can Contribute to Rapid and Massive Diagnostics of Respiratory Virus Infections: COVID-19 Case. ACS Sensors 5, 26632678

61 Mandal, N. et al. (2021) PSA detection using label free graphene FET with coplanar electrodes based microfluidic point of care diagnostic device. Talanta 222, 121581

62 Chen, S. et al. (2020) Donor effect dominated molybdenum disulfide/graphene nanostructure-based field-effect transistor for ultrasensitive DNA detection. Biosens. Bioelectron. 156,112128

63 Danielson, E. et al. (2020) Graphene based field-effect transistor biosensors functionalized using gas-phase synthesized gold nanoparticles. Sensors Actuators, B Chem. 320, 128432

64 Mensah, K. et al. (2020) DNA Hybridization Measured with Graphene Transistor Arrays. Adv. Healthc. Mater. 9, 2000260

65 Ganguli, A. et al. (2020) High Sensitivity Graphene Field Effect Transistor-Based Detection of DNA Amplification. Adv. Funct. Mater. 30, 1-9

66 Hwang, M.T. et al. (2020) Ultrasensitive detection of nucleic acids using deformed graphene channel field effect biosensors. Nat. Commun. 11, 1543

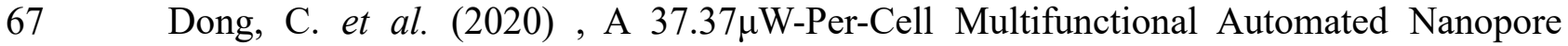
Sequencing CMOS Platform with 16*8 Biosensor Array., pp. 1-4

$68 \mathrm{Xu}, \mathrm{H}$. et al. (2018) Effects of Nanomaterial Saturable Absorption on Passively ModeLocked Fiber Lasers in an Anomalous Dispersion Regime: Simulations and Experiments. IEEE J. Sel. Top. Quantum Electron. 24,

69 van Belkum, A. and Rochas, O. (2018) Laboratory-Based and Point-of-Care Testing for MSSA/MRSA Detection in the Age of Whole Genome Sequencing. Front. Microbiol. 9, 1437

70 Yu, Y.S. et al. (2018) Computational investigation on DNA sequencing using functionalized graphene nanopores. Phys. Chem. Chem. Phys. 20, 9063-9069

71 Garaj, S. et al. (2013) Molecule-hugging graphene nanopores. Proc. Natl. Acad. Sci. U. S. A. 110, 12192-12196

72 Wells, D.B. et al. (2012) Assessing graphene nanopores for sequencing DNA. Nano Lett. $12,4117-4123$

73 Shankla, M. and Aksimentiev, A. (2019) Step-defect guided delivery of DNA to a graphene nanopore. Nat. Nanotechnol. 14, 858-865

74 Zou, A. et al. (2020) Spontaneous Translocation of Single-Stranded DNA in GrapheneMoS 2 Heterostructure Nanopores: Shape Effect. J. Phys. Chem. B DOI: 10.1021/acs.jpcb.0c06934

$75 \mathrm{Li}$, J. et al. (2017) The impact of the number of layers of the graphene nanopores and the electrical field on ssDNA translocation. Mol. Simul. 43, 320-325

76 Bollella, P. et al. (2017) Beyond graphene: Electrochemical sensors and biosensors for biomarkers detection, 89Elsevier.

77 Ray, S. et al. (2018) Design of Ultrasensitive Protein Biosensor Strips for Selective Detection of Aromatic Contaminants in Environmental Wastewater. Anal. Chem. 90, 8960-8968

78 Zhurauski, P. et al. (2018) Sensitive and selective Affimer-functionalised interdigitated electrode-based capacitive biosensor for Her4 protein tumour biomarker detection. Biosens. Bioelectron. 108, 1-8

79 Rauf, S. et al. (2018) Carboxylic group riched graphene oxide based disposable electrochemical immunosensor for cancer biomarker detection. Anal. Biochem. 545, 13-19 
80 Panikar, S.S. et al. (2020) Anti-fouling SERS-based immunosensor for point-of-care detection of the B7-H6 tumor biomarker in cervical cancer patient serum. Anal. Chim. Acta 1138, $110-122$

81 Wei, B. et al. (2018) Graphene nanocomposites modified electrochemical aptamer sensor for rapid and highly sensitive detection of prostate specific antigen. Biosens. Bioelectron. 121, 4146

82 Haslam, C. et al. (2018) Label-Free Sensors Based on Graphene Field-Effect Transistors for the Detection of Human Chorionic Gonadotropin Cancer Risk Biomarker. Diagnostics 8, 5

83 Fan, Y. et al. (2019) A paper-based electrochemical immunosensor with reduced graphene oxide/thionine/gold nanoparticles nanocomposites modification for the detection of cancer antigen 125. Biosens. Bioelectron. 135, 1-7

84 Bharti, A. et al. (2020) An electrochemical aptasensor for analysis of MUC1 using gold platinum bimetallic nanoparticles deposited carboxylated graphene oxide. Anal. Chim. Acta 1097, $186-195$

85 Morgado, M. et al. (2016) Tumor necrosis factor- $\alpha$ and Interferon- $\gamma$ stimulate MUC16 (CA125) expression in breast, endometrial and ovarian cancers through NFKB. Oncotarget 7, $14871-14884$

86 Seo, G. et al. (2020) Rapid Detection of COVID-19 Causative Virus (SARS-CoV-2) in Human Nasopharyngeal Swab Specimens Using Field-Effect Transistor-Based Biosensor. ACS Nano 14, 5135-5142

87 Staiano, M. et al. (2017) Enzymes as Sensors. In Methods in Enzymology 589pp. 115-131, Academic Press

88 Eggins, B. (2013) Biosensors: An Introduction, Springer-Verlag212.

89 Mehrotra, P. Biosensors and their applications - A review. , Journal of Oral Biology and Craniofacial Research, 6. 01-May-(2016) , Elsevier, 153-159

90 Navaee, A. and Salimi, A. (2018) FAD-based glucose dehydrogenase immobilized on thionine/AuNPs frameworks grafted on amino-CNTs: Development of high power glucose biofuel cell and biosensor. J. Electroanal. Chem. 815, 105-113

91 Ispas, C.R. et al. Review: Recent Developments in Enzyme-Based Biosensors for Biomedical Analysis. , Analytical Letters, 45. (2012) , 168-186

92 Hajian, R. et al. (2019) Detection of unamplified target genes via CRISPR-Cas9 immobilized on a graphene field-effect transistor. Nat. Biomed. Eng. 3, 427-437

93 Xuan, X. et al. (2018) A wearable electrochemical glucose sensor based on simple and lowcost fabrication supported micro-patterned reduced graphene oxide nanocomposite electrode on flexible substrate. Biosens. Bioelectron. 109, 75-82

94 Al-Sagur, H. et al. (2019) Amperometric glucose biosensing performance of a novel graphene nanoplatelets-iron phthalocyanine incorporated conducting hydrogel. Biosens. Bioelectron. 139, 111323

95 Maity, D. et al. (2019) Glucose oxidase immobilized amine terminated multiwall carbon nanotubes/reduced graphene oxide/polyaniline/gold nanoparticles modified screen-printed carbon electrode for highly sensitive amperometric glucose detection. Mater. Sci. Eng. C 105, 110075

$96 \mathrm{Xu}$, B. et al. (2020) Graphene oxide-functionalized long period fiber grating for ultrafast label-free glucose biosensor. Mater. Sci. Eng. C 107, 110329

$97 \mathrm{Lu}, \mathrm{Z}$. et al. (2021) Novel flexible bifunctional amperometric biosensor based on laser engraved porous graphene array electrodes: Highly sensitive electrochemical determination of hydrogen peroxide and glucose. J. Hazard. Mater. 402, 123774 
98 Martínez-García, G. et al. (2017) An electrochemical enzyme biosensor for 3hydroxybutyrate detection using screen-printed electrodes modified by reduced graphene oxide and thionine. Biosensors 7, 50

99 Patel, S.K.S. et al. (2017) Eco-friendly composite of Fe3O4-reduced graphene oxide particles for efficient enzyme immobilization. ACS Appl. Mater. Interfaces 9, 2213-2222

100 Povedano, E. et al. (2017) Decoration of reduced graphene oxide with rhodium nanoparticles for the design of a sensitive electrochemical enzyme biosensor for $17 \beta$-estradiol. Biosens. Bioelectron. 89, 343-351

101 Patel, S.K.S. et al. (2018) Fe2O3 yolk-shell particle-based laccase biosensor for efficient detection of 2,6-dimethoxyphenol. Biochem. Eng. J. 132, 1-8

102 López Marzo, A.M. et al. (2020) 3D-printed graphene direct electron transfer enzyme biosensors. Biosens. Bioelectron. 151, 111980

103 Wehling, T.O. et al. (2008) Molecular Doping of Graphene. Nano Lett. 8, 173-177

104 Zhang, A. and Lieber, C.M. Nano-Bioelectronics. Chemical Reviews, 116. (2016), 215257

105 Hwang, H.M. et al. (2016) Mesoporous Non-stacked Graphene-receptor Sensor for Detecting Nerve Agents. Sci. Rep. 6,

106 MacChia, E. et al. About the amplification factors in organic bioelectronic sensors. Materials Horizons, 7. (2020), 999-1013

107 Rivnay, J. et al. (2018) Organic electrochemical transistors. Nat. Rev. Mater. 3, 17086

108 Li, H. et al. (2017) Graphene field effect transistors for highly sensitive and selective detection of K+ ions. Sensors Actuators, B Chem. 253, 759-765

109 Mackin, C. et al. (2018) Chemiresistive Graphene Sensors for Ammonia Detection. ACS Appl. Mater. Interfaces 10, 16169-16176

110 Wicaksono, D.H.B. et al. (2018), Preliminary study on graphene/metal oxide nanoparticles-coated cotton fabrics for flexible gas sensor. , in AIP Conference Proceedings, 2024, pp. 020063

111 He, L. et al. (2017) Label-free femtomolar cancer biomarker detection in human serum using graphene-coated surface plasmon resonance chips. Biosens. Bioelectron. 89, 606-611

112 Singh, M. et al. (2019) Ultra-Sensitive Fiber Optic Gas Sensor Using Graphene Oxide Coated Long Period Gratings. IEEE Photonics Technol. Lett. 31, 1473-1476

113 Di Gilio, A. et al. (2020) Breath Analysis for Early Detection of Malignant Pleural Mesothelioma: Volatile Organic Compounds (VOCs) Determination and Possible Biochemical Pathways. Cancers (Basel). 12, 1262

114 Traxler, S. et al. (2018) VOC breath profile in spontaneously breathing awake swine during Influenza A infection. Sci. Rep. 8,

115 Kohlmuller, D. and Kochen, W. (1993) Is n-Pentane Really an Index of Lipid Peroxidation in Humans and Animals? A Methodological Reevaluation. Anal. Biochem. 210, 268-276

116 Chen, W. et al. (2016) Biochemical pathways of breath ammonia (NH3) generation in patients with end-stage renal disease undergoing hemodialysis. J. Breath Res. 10, 036011

117 Bhardwaj, S.K. et al. (2018) A three-phase copper MOF-graphene-polyaniline composite for effective sensing of ammonia. Anal. Chim. Acta 1043, 89-97

$118 \mathrm{Wu}, \mathrm{G}$. et al. (2018) Simulation of Graphene Field-Effect Transistor Biosensors for Bacterial Detection. Sensors 18, 1715

119 Yadegari, A. et al. (2017) An electrochemical cytosensor for ultrasensitive detection of cancer cells using modified graphene-gold nanostructures. RSC Adv. 7, 2365-2372 
120 Chowdhury, A.D. et al. (2018) Impedimetric biosensor for detection of cancer cells employing carbohydrate targeting ability of Concanavalin A. Biosens. Bioelectron. 122, 95-103

121 Zhang, H. et al. (2016) Rapid and Sensitive Detection of Cancer Cells Based on the Photothermal Effect of Graphene Functionalized Magnetic Microbeads. ACS Appl. Mater. Interfaces 8, 29933-29938

122 Thakur, B. et al. (2018) Rapid detection of single E. coli bacteria using a graphene-based field-effect transistor device. Biosens. Bioelectron. 110, 16-22

123 Pandey, A. et al. (2017) Graphene-interfaced electrical biosensor for label-free and sensitive detection of foodborne pathogenic E. coli O157:H7. Biosens. Bioelectron. 91, 225-231

124 Kim, K.H. et al. (2020) High-performance portable graphene field-effect transistor device for detecting Gram-positive and -negative bacteria. Biosens. Bioelectron. 167, 112514

125 Hampitak, P. et al. (2020) Protein interactions and conformations on graphene-based materials mapped using quartz-crystal microbalance with dissipation monitoring (QCM-D). Carbon N. Y. 165, 317-327

126 Hampitak, P. et al. (2020) A Point-of-Care Immunosensor Based on a Quartz Crystal Microbalance with Graphene Biointerface for Antibody Assay. ACS Sensors DOI: 10.1021/acssensors.0c01641

127 Kaushik, A.K. et al. (2020) Electrochemical SARS-CoV-2 Sensing at Point-of-Care and Artificial Intelligence for Intelligent COVID-19 Management. ACS Appl. Bio Mater. DOI: 10.1021/acsabm.0c01004

128 Shrivastava, S. et al. Recent progress, challenges, and prospects of fully integrated mobile and wearable point-of-care testing systems for self-testing. , Chemical Society Reviews, 49. 21Mar-(2020), Royal Society of Chemistry, 1812-1866

$129 \mathrm{Li}$, S. et al. (2019) Electrogenerated chemiluminescence on smartphone with graphene quantum dots nanocomposites for Escherichia Coli detection. Sensors Actuators, B Chem. 297, 126811

130 Aspermair, P. et al. (2020) Reduced graphene oxide-based field effect transistors for the detection of E7 protein of human papillomavirus in saliva. Anal. Bioanal. Chem. DOI: $10.1007 / \mathrm{s} 00216-020-02879-\mathrm{z}$ 